\title{
Contralateral paradoxical response to chemotherapy in tuberculous pleural effusion
}

\author{
AlaEldin Hassan Ahmed ${ }^{1,2}$ and Sarah Goddard ${ }^{2}$
}

\section{Introduction}

Pleural effusions may occur as a complication of primary tuberculosis or an established pulmonary or extrapulmonary infection. New formation or expansion of a tuberculous lesion during chemotherapy is referred to as paradoxical response. Paradoxical response has been described to occur weeks or months after starting antituberculous chemotherapy for parenchymal lung disease, intracranial tuberculoma, or pleural effusion $^{1-2}$.

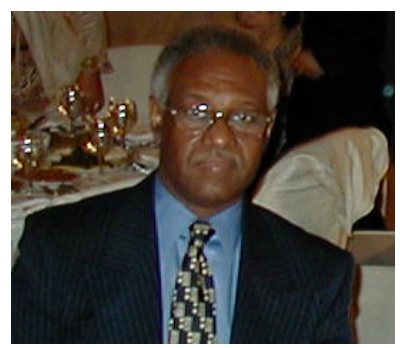

To our knowledge only one case of contralateral paradoxical effusion that occurred eight weeks after standard antituberculous chemotherapy has been reported in the literature ${ }^{3}$. In this paper, we describe a patient who developed contralateral paradoxical pleural effusion six days after standard antituberculous chemotherapy and prednisolone.

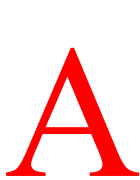

Case Report

23-year Asian lady was admitted to our ward (City General Hospital, Stoke-on-Trent) with a three weeks history of shortness of breath on moderate exertion, cough productive of white sputum, night sweats, and one stone weight loss. Clinical examination was normal except for signs of left pleural effusion; this was confirmed on X-ray of the chest (plate 1). The full blood count showed haemoglobin of 8.9 $\mathrm{g} / \mathrm{dl}$, white blood cell count $6.2 \times 10^{9} / \mathrm{l}$, mean corpuscular volume $53.9 \mathrm{fl}$, mean corpuscular haemoglobin 17.1 pg (patient is known thalassaemia trait). The ESR was $55 \mathrm{~mm} / \mathrm{hr}$ and the $\mathrm{C}$ - reactive protein was raised at $171 \mathrm{mg} / \mathrm{l}$. At thoracocentesis straw coloured fluid was aspirated. The fluid was an exudate: protein content was $56 \mathrm{~g} / \mathrm{l}$. The Gram stain was negative. A pleural biopsy obtained at the time of thoracocentesis, showed several foci of caseous necrosis surrounded by palisaded histeocytes with scattered langhans cells; Ziehl-Nielsen staining was positive for acid fast bacilli.

1. Department of Medicine, University of Khartoum, Khartoum, Sudan.

2. Department of Respiratory Medicine, City General Hospital, Stoke-on-Trent, ST4 6QG, UK

Correspondence to Dr. AlaEldin H. Ahmed, Department of Medicine, University of Khartoum, Khartoum, Sudan
The pleural effusion was drained to dryness and the patient was started on standard antituberculous treatment with rifampicin, isoniazid, and pyrazinamide, in addition to pyridoxine and prednisolone $30 \mathrm{mgs}$ per day.

The patient improved clinically and her temperature was normal before she was discharged home to continue on the same medications.

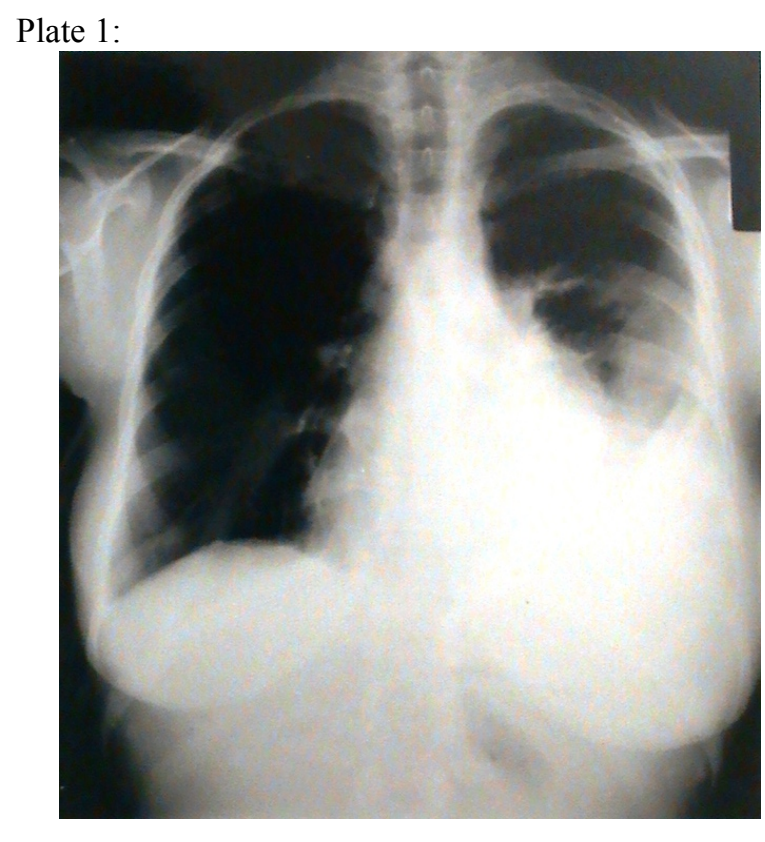

Although chemotherapy was not interrupted at any time, the patient was readmitted three days after discharge (six days after initiation of anti tuberculous therapy) with feeling cold and shivery and had right 
side pleuritic chest pain and headache. Physical examination revealed: temperature raised at $38^{\circ} \mathrm{C}$ and there were signs of right side pleural effusion which was confirmed on X-ray of the chest (plate 2). Full blood count had not changed. Liver function test showed a slightly raised Gamma Glutamyl transferase at $38 \mathrm{U} / \mathrm{L}$ (normal 0 - 35), and the CRP was 178 $\mathrm{mg} / \mathrm{l}$.

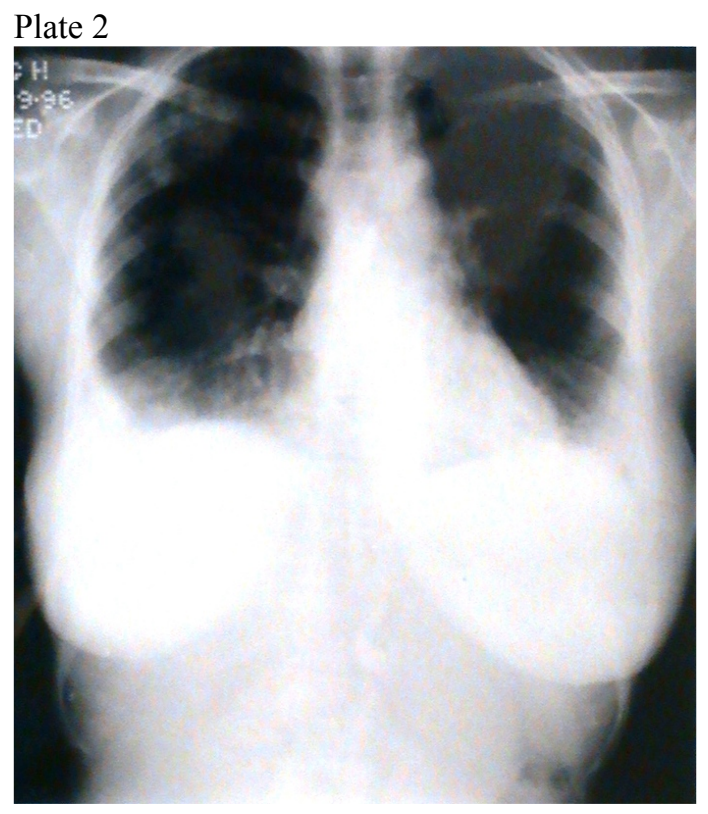

Atituberculous chemotherapy and prednisolone were continued. Thoracocentesis was not performed on this admission. The patient continued to have high temperature for six days; this eventually settled and she was discharged home after seven days of hospitalization on rifampicin, isoniazid, pyrazinamide, pyridoxine and $30 \mathrm{mg}$ prednisolone. The prednisolone was continued for further four weeks and stopped after gradual reduction over 20 days. The patient was followed-up in clinic and after two months of treatment the chest X-ray was normal (plate 3).

\section{Discussion:}

Modern chemotherapy regimens for the treatment of tuberculosis are associated with high level of effectiveness and minimal side effects $^{4}$. Paradoxical worsening of pleural effusions has, however, been described ${ }^{1-3,5}$. A survey reported an incidence of $16 \%$ among 61 patients with tuberculous pleural effusions ${ }^{5}$.
The mechanisms by which paradoxical response occur are speculative.

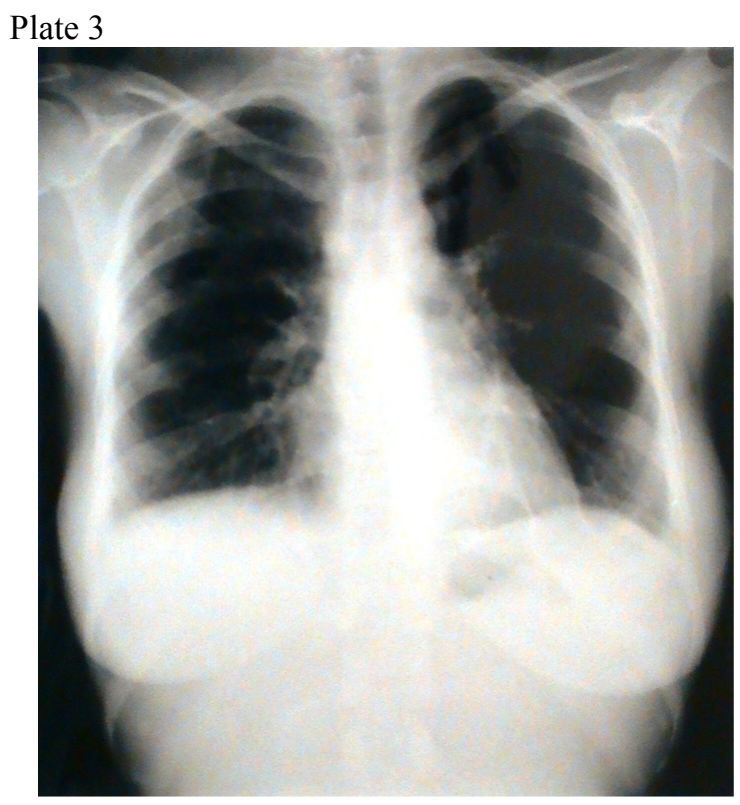

In the past such response was thought to result from rupture into the pleural space of subpleural caseous foci, mediastinal lymph nodes, or haematogenous dissemination, and mycobacterium was cultured from pleural fluid several weeks after initiation of therapy. In recent years, however, this response is thought to represent local manifestations of heightened delayed hypersensitivity mounted against immunogenic cell wall substances resealed from dying tuberculous bacilli in patients whose level of cellular immunity is enhanced as a result of chemotherapy ${ }^{6}$.

Two points, however, deserve special discussion in this case. Firstly, contralateral effusion developed only six days after initiation of treatment whereas a previous case report has shown this to occur many weeks after starting therapy ${ }^{3}$. It would, therefore, be important to consider such a reaction in the differential diagnosis of patients who develop new symptoms soon after initiation of antituberculous chemotherapy. Secondly, our patient was taking prednisolone when she developed the paradoxical pleural effusion. Although steroids are known to modify the severity of clinical manifestations of tuberculosis, it has clearly failed to prevent the paradoxical response in this patient.

Interestingly, the only case of paradoxical 
response reported in the literature was also taking prednisolone ${ }^{3}$. What we can not be certain of is whether the prednisolone has reduced the severity of reaction in these two patients. Indeed, the pleural effusion that developed in the patient we report was not large and required no drainage. In the series reported by Al-Majed ${ }^{5}$ six of ten patients who developed paradoxical worsening of their effusion had respiratory distress with massive effusions requiring pleural aspirations; none of these patients were taking steroids at the time of presentation. Clearly a prospective placebocontrolled study to address this issue is warranted.

\section{References}

1. Cheng VC, Ho PL, Lee RA, et al. Clinical spectrum of paradoxical deterioration during antituberculosis therapy in non-HIV infected patients. Eur $J$ Clin Microbiol Infect Dis 2002;21(11):803-9.

2. Breen RAM, Smith CJ, Bettinson $\mathrm{H}$, et al. Paradoxical reaction during tuberculosis treatment in patients with and without HIV co-infection. Thorax 2004; 59:704-7.

3. Vilaseca J, Lopez-Vivancos J, Arnau J, et al. Contralateral pleural effusion during chemotherapy for tuberculous pleurisy. Tubercle 1984;65:209-10.

4. CDC, American thoracic society and infectious disease society of America. Treatment of tuberculosis. MMWR 2003;52(RR11):1-77.

5. Al-Majed SA. Study of paradoxical response to chemotherapy in tuberculous pleural effusion. Respir Med 1996;90:211-14.

6. Cheng VC, Yuen KY, Chan WM, et al. Immuno restitution disease involving the innate and adaptive response. Clin Infect Dis 2000;30(6):882-92. 